\title{
ENTRE LA IMPOSIBLE ACOGIDA Y LA IRREMISIBLE EXISTENCIA. HACIA UNA FENOMENOLOGÍA DEL SUFRIMIENTO EN LÉVINAS
}

\author{
Between the impossible reception and the irremissible existence. \\ Towards a phenomenology of suffering in Lévinas \\ Bryan Zúñiga Iturra \\ Universidad de Chile, Santiago, Chile \\ bryan.zuniga@ug.uchile.cl
}

\begin{abstract}
Resumen
El presente artículo tiene como objetivo principal dejar de manifiesto algunas notas fenomenológicas acerca del sufrimiento, teniendo como hilo conductor algunas de las ideas elaboradas por Emmanuel Lévinas. De este modo, mi intención en este texto es responder a la pregunta ¿cómo se manifiesta el sufrimiento a quiénes lo padecen? Así, con la finalidad de resolver esta inquietud este trabajo se estructura en dos momentos. En primer lugar, y siguiendo los planteamientos presentados en La souffrance inutile, estudiaré la dimensión corporal y sensible a través de la que se revela esta experiencia. En segundo lugar, y continuando la reflexión trazada por el autor en Le temps et l'autre y Totalité et infini, analizaré el carácter irremisible mediante el que nos aparece esta vivencia. Para concluir pondré en evidencia algunas de las principales notas del sufrimiento que deja de manifiesto esta indagación.
\end{abstract}

Palabras clave: sufrimiento, acogida, irremisible, sensibilidad, existencia.

\begin{abstract}
The main objective of this article is to reveal some phenomenological notes about suffering, having as a thread some of the ideas elaborated by Emmanuel Lévinas. In this way, my intention in this work is to answer the following question: How does suffering manifest itself to those who suffer from it? So, in order to solve this concern my article is structured in two moments. First, and following the approaches sketched by the author presented in La souffrance inutile, I will study the body and sensitive dimension through which this experience is revealed. Secondly, and continuing the reflection maked by Lévinas in Le temps et l'autre and Totalité et infini, I will analyse the irremissible character through which this experience appears to us. In conclusion, I will highlight some of the main notes of suffering that this work reveals.
\end{abstract}

Keywords: suffering, reception, irremissible, sensibility, existence.

Fecha de Recepción: 05/05/2019 - Fecha de Aceptación: 18/07/2019 


\section{Introducción}

En su texto El tiempo, tribunal de la historia, Manuel Reyes Mate establece lo siguiente: “El concepto de tiempo está ligado al del sufrimiento [...] ese tiempo que inaugura el primer Adán pone en marcha una historia destinada a encontrar una respuesta a ese sufrimiento que será coronada por el segundo Adán” (2018 19). Si seguimos el pasaje recién citado, podemos afirmar que desde sus orígenes la historia de la humanidad ha ido acompañada por distintas figuraciones del sufrimiento a las que ésta ha dado lugar, como, por ejemplo, el hambre y la pobreza padecida diariamente por gran parte de las personas que habitan nuestro planeta, o bien, las así denominadas "enfermedades mentales", mediante las que se miden los padecimientos espirituales de los sujetos de acuerdo a los parámetros de la ciencia médica. De este modo, pareciera ser que cuando queremos hablar y reflexionar acerca de la historia humana, el sufrimiento resulta un pasaje ineludible, razón por la que el intento de abordar esta experiencia por medio de una óptica fenomenológica -en tanto estudio de nuestras diferentes vivencias-, adquiere una relevancia indesmentible.

Teniendo presente esta descripción preliminar, el presente artículo tiene como objetivo principal dejar de manifiesto algunas notas fenomenológicas acerca del sufrimiento, teniendo como hilo conductor algunas de las ideas elaboradas por Emmanuel Lévinas, así como su discusión con la teoría fenomenológica de la atención elaborada por Edmund Husserl. De este modo, el siguiente texto poniendo entre paréntesis las causas fisiológicas, psicológicas o históricas de esta experiencia, busca responder a la pregunta ¿cómo se manifiesta el sufrimiento a aquéllos y aquéllas que lo padecen en cuerpo propio? Con la finalidad de resolver esta inquietud mi artículo se estructura en dos momentos. En primer lugar, y siguiendo los planteamientos bosquejados por el autor en La souffrance inutile, estudiaré la dimensión corporal y sensible a través de la que se revela esta experiencia, o sea, el tipo de intencionalidad -o bien la ausencia de ella- por medio de la que tiene lugar este acontecimiento. En segundo lugar, y continuando la reflexión trazada por Lévinas en Le temps et l'autre y Totalité et infini, analizaré el carácter irremisible mediante el cual nos aparece esta vivencia, esto es, la dimensión ineludible que ella comporta para quien la padece. Para concluir pondré en evidencia algunas de las principales notas del sufrimiento que deja de manifiesto este trabajo. 


\section{De la imposible acogida. El sufrimiento como fractura del modelo intencional de la conciencia}

En su texto La souffrance inutile, Lévinas intentando dar cuenta de la dimensión sensible mediante la que se revela esta experiencia, la describe a partir de su carácter inasumible, es decir, por medio del exceso que hace imposible toda recepción de este acontecimiento para quien lo evidencia. En otros términos, el autor buscando establecer la dimensión corporal de esta vivencia, se encuentra con que ella tiene como soporte un tipo específico de sensación que desborda toda capacidad subjetiva que intente acogerla (acolligere), o sea, admitirla o integrarla dentro de su horizonte de sentido.

De este modo, el sufrimiento se presenta de entrada como una experiencia que supera absolutamente nuestra sensibilidad y, con ello, toda posible capacidad de síntesis que busque agrupar $u$ ordenar los diversos datos corporales a través de los que se torna patente.

Realizada esta primera aproximación, dos de las inquietudes filosóficas a la que nos remite la imposibilidad de acoger esta vivencia son precisamente 1) ¿qué significa que una subjetividad acoja o reciba un acontecimiento determinado? y 2) ¿a qué se refiere el autor cuando establece que la experiencia del sufrimiento mienta un tipo específico de sensación? Con la finalidad de resolver ambas interrogantes es menester hacer referencia a la teoría fenomenológica de la atención con la que discute Lévinas, a saber, aquella presentada por Edmund Husserl en su obra Experiencia y juicio con la intención de dar cuenta de las estructuras receptivas de la conciencia. De esta manera, y teniendo a la vista la misión de este texto, abordaré esquemáticamente la doctrina husserliana acerca de la atención y, con ello, la comprensión fenomenológica de la sensación contenida en ésta.

Así las cosas, y siguiendo el orden de la argumentación esbozada por Husserl, responderé en primer lugar la segunda inquietud presentada previamente, esta es, aquella relativa al estatuto fenomenológico de la sensación, para de manera posterior resolver la primera pregunta, a saber, aquella que remite a la capacidad de recepción o acogida de parte de la subjetividad.

Pues bien, cuando hablamos de sensación en el marco de la teoría husserliana de la atención hacemos referencia a su célebre doctrina de la asociación, es decir, a aquella que mienta aquella síntesis pasiva por medio de la cual se estructura nuestro campo sensible, o sea, las diferentes sensaciones que, despertando nuestro interés posibilitan que nos volvamos sobre ellas de modo intencional, vale decir, mediante el esquema conciencia-de -una determinada sensación-. En otros términos, es a partir de este tipo de síntesis -que en tanto pasiva se produce a espaldas del yo- que se constituyen y organizan los diversos 
momentos sensibles que nos afectan, es decir que motivan nuestro interés, permitiendo de esta forma que tomemos conciencia teórica de ellos. De esta manera, tal como sostiene Andrés Osswald el campo sensible abarca a la totalidad del relieve afectante de nuestra experiencia (2017 75-76) y sólo a partir de él resulta posible nuestra actividad intencional. Dicho de otro modo, sin la constitución pasiva de la sensación no habría afección y, con ello, despertar intencional de la conciencia.

Siguiendo este hilo conductor, cuando hablamos de atención en el contexto de Experiencia y juicio nos referimos a aquel momento de actividad de la conciencia en la que ésta se vuelve sobre aquellos datos sensibles agrupados de modo pasivo mediante la asociación. En otra formulación, si la asociación mienta la constitución de las diversas materias sensibles que despiertan nuestro interés, vale decir que nos afectan, por su parte, la atención corresponde a aquel momento en el que nos volvemos aprehensivamente sobre las primeras, destacando de esta manera los diferentes aspectos y objetos que captamos en nuestras vivencias. En el parágrafo dieciocho de Experiencia y juicio podemos leer lo siguiente:

En general, la atención es una tendencia del yo hacia el objeto intencional, hacia la unidad que continuamente aparece en el cambio de modo del dato; es una tendencia realizadora que pertenece a la estructura esencial de un acto específico del yo (de un acto del yo en el sentido estricto del término). La realización que se inicia con la orientación-hacia, el punto inicial de la realización del acto, constituye el comienzo de un estar-dirigido continuo y realizador, del yo hacia el objeto. (1980 87)

De acuerdo con esto, el fenómeno de la atención representa aquel momento de nuestra experiencia en el que comienza nuestra actividad intencional, vale decir, aquel volverse activo sobre las diferentes sensaciones que al afectarnos motivan nuestro interés. En este sentido, Husserl denomina a este primer nivel de actividad de la conciencia receptividad en cuanto mienta la capacidad subjetiva de acoger de una determinada manera la afección por medio de la cual un determinado objeto convoca nuestra mirada intencional (1980 86). En otras palabras, la atención es recepción y acogida en la medida que a la sensación que nos afecta pasivamente sigue un momento de actividad en el que respondiendo a dicho estímulo nos volvemos sobre él de un modo preciso, constituyéndolo, por ejemplo, como aquel fenómeno que se manifiesta en tal o cual perspectiva o posee determinada intensidad $^{1}$.

\footnotetext{
${ }^{1}$ Con relación a este punto, Husserl describe el carácter constituyente de la subjetividad de la siguiente manera: "me he descubierto a mí mismo como el ego trascendental que constituye en su vida constituidora todo lo que me es objetivo; el yo de todas las constituciones" (2005 160). Así, bajo este prisma la subjetividad es descrita de entrada a partir de su capacidad de otorgar sentido a
} 
Si queremos resumir este punto $y$, con ello esta doctrina, podemos afirmar que cuando hablamos de acogida y recepción en el marco de la propuesta husserliana dos son los elementos que tenemos en mente. Primero, un momento pasivo en el que un sujeto es afectado o despertado sensiblemente por un fenómeno particular, como por ejemplo, cuando nos damos cuenta tras una larga jornada que nuestro cuerpo se encuentra agotado. Segundo, un momento activo en el que dicha subjetividad despertada pasivamente por una experiencia particular se vuelve sobre ésta de modo intencional, vale decir, toma conciencia teórica de ella. De esta forma, si en un primer instante, la receptividad implica la posibilidad de ser afectados a nuestro pesar por los diferentes sucesos que evidenciamos, al mismo tiempo dicha experiencia pasiva se posiciona como el fundamento de un tipo de actividad mediante la que ordenamos y sintetizamos los diversos datos sensibles que captamos, otorgándoles así un sentido o significación precisa que nos permite conocerlos. En otras palabras, este tipo particular de actividad en la pasividad en el que consiste la receptividad ${ }^{2}$, mienta el poder que tiene la

todas aquellas vivencias sobre las que se vuelve de modo intencional. De esta forma, la 'teoría de la atención' desarrollada por el autor cumple un rol filosófico fundamental en su fenomenología, pues si la subjetividad es definida en virtud de su actividad constituyente, aquella doctrina que mienta las condiciones de dicha constitución adquiere una relevancia metodológica indesmentible. En razón de esto, una propuesta filosófica que busque cuestionar dicho planteamiento -que como veremos es el caso de aquella teoría desarrollada por Lévinas en sus análisis acerca del sufrimiento, viene a poner en tensión todas aquellas bases filosóficas que permiten describir a la subjetividad mediante su carácter constituyente. En otras palabras, quien cuestiona la doctrina husserliana de la atención, cuestiona, en último término, su descripción de la subjetividad como una constituyente o trascendental.

${ }^{2}$ En la presentación de su teoría de la 'recepción' elaborada en Experiencia y juicio, Husserl establece que: "Según esto, no existe únicamente una pasividad previa a la actividad, como pasividad del fluir temporal, originariamente constitutivo, aunque sólo pre-constitutivo, sino también una pasividad superpuesta, propiamente objetivadora, es decir, que tematiza o co-tematiza los objetos; una pasividad que pertenece al acto no como base, sino como acto, una especie de pasividad en la actividad." (1980 117). De acuerdo con esto, el autor distingue entre dos tipos de pasividad; de una parte, tenemos aquella pasividad que es 'previa' a la actividad constituyente de la subjetividad, como es el caso de aquella recogida en su doctrina de la 'asociación', es decir, aquella que mienta la constitución pasiva de las diferentes sensaciones que experimentamos y; de otra, tenemos una forma de pasividad que tiene lugar 'en' el seno mismo de nuestra actividad intencional, como es el caso de aquella referida en la doctrina husserliana acerca de la atención, donde nos encontramos con una subjetividad que, al tiempo que es afectada pasivamente por una determinada sensación, se vuelve sobre ésta de modo intencional. Así las cosas, algunas de las preguntas que naturalmente pueden surgir a partir de esta exposición son las siguientes: 1) ¿alguna de estas formas de pasividad descritas por el autor, nos permiten comprender el fenómeno del sufrimiento? y 2) ¿es que acaso esta experiencia nos revela un modo de pasividad más potente que aquellas conceptualizadas por Husserl? En la segunda parte de este artículo abordaremos la noción levinasiana de 'pasividad radical' mediante la que este autor describe el sufrimiento. 
conciencia para imprimir una forma determinada a todo aquello que experimenta, posibilitando de esta manera que ésta se vuelva intencionalmente sobre todas sus vivencias.

En este sentido, y siguiendo el significado etimológico de la palabra, podemos sostener que recibir (re-capere) es capturar, tomar, es decir, imponer aquellas condiciones subjetivas que permiten hacer conocimiento de todo lo que nos aparece. Con la finalidad de aclarar aun más este esquema, podemos pensar en un objeto concreto de nuestro campo sensible, como por ejemplo, el computador que utilizo en este momento para escribir mi texto. Si analizamos esta experiencia de acuerdo a la teoría husserliana de la receptividad, podemos sostener que, en un primer instante, este objeto despierta pasivamente mi atención, lo que permite a su vez que me dirija intencionalmente sobre el mismo, haciendo conocimiento de él como aquel computador que veo en tal o cual perspectiva o que posee determinada significación en función de cómo se presenta sensiblemente para mí.

Pero, ¿acaso es aplicable este modelo explicativo en el caso del sufrimiento? Lévinas responde negativamente ante esta inquietud, afirmando en el ensayo antes referido que en el sufrimiento la conciencia "no opera como toma de conciencia, sino como revulsión" (1991 101) ${ }^{3}$. Esto viene a significar que este fenómeno pone en tensión todas aquellas descripciones de la corporalidad que la comprenden a la luz de la actividad teórica de la conciencia, en la medida que en este acontecimiento si hay algo que resulta imposible para quien lo padece es precisamente hacer conocimiento de esta experiencia. Dicho de otra manera, hacia el sufrimiento no podemos volvernos como si fuese un objeto a conocer, pues la estructura misma del volverse de la conciencia, es decir, la intencionalidad, se encuentra fuera de juego en este suceso.

Así, si intentamos reconstruir esta vivencia, podemos sostener que en ella el esquema conciencia intencional-objeto intencionado ${ }^{4}$ resulta inaplicable pues, ¿qué

3 "non comme 'prise', mais comme révulsion". En lo sucesivo, las obras citadas cuyo idioma original es el francés, han sido traducidas por el autor de este artículo.

4 En el parágrafo treinta y cinco de sus Ideas relativas a una fenomenología pura y una filosofía fenomenológica (Ideas I) Husserl señala lo siguiente: "En general es inherente a la esencia de todo cogito actual ser conciencia de algo. pero según lo antes expuesto, la cogitatio modificada es a su modo igualmente conciencia, y de lo mismo que la correspondiente no modificada (...) Todas las vivencias que tienen en común estas propiedades esenciales se llaman también 'vivencias intencionales'; en tanto que son conciencia de algo, se dicen 'referidas intencionalmente' a este algo" (2013 154). De acuerdo con este pasaje, uno de los principios fundamentales de la fenomenología husserliana es la descripción de todas nuestras vivencias a partir de su carácter 'intencional', es decir, mediante el 'volverse constituyente' de la subjetividad sobre sus diferentes experiencias. En otros términos, para el autor todas nuestras vivencias tienen lugar por medio del modelo 'conciencia intencional'-'objeto intencionado', motivo por el que, desde esta perspectiva 
objeto intencional encontramos allí donde la conciencia misma es excedida absolutamente al punto de desgarrarse? Si resolvemos esta cuestión siguiendo a Lévinas, podemos sostener que este padecimiento conmociona a tal nivel las capacidades cognitivas de la subjetividad que en ella se halla ausente toda posible forma de conciencia. En otros términos, la afección por medio de la que se presenta el sufrimiento posee tal magnitud que fractura la estructura misma de la receptividad, vale decir, aquella que permite a un sujeto acoger las diferentes sensaciones que experimenta.

De este modo, una subjetividad sufriente es en primera instancia, aquella cuya pretendida capacidad de dar sentido a todas sus vivencias es puesta en tensión por esta experiencia que la atrapa. Lévinas nos dice: “Que en su fenómeno propio, intrínsecamente, el sufrimiento sea inútil, que él sea 'para nada', es por tanto, lo menos que podemos decir de él" (1991 102) ${ }^{5}$. Teniendo a la vista esta descripción, podemos sostener que el sufrimiento es inútil, o sea, carece de significación en la medida que esta experiencia corporal conmociona en tal grado nuestro poder de conocimiento que, incluso inmoviliza nuestra propia capacidad de encontrarle un sentido cuando lo padecemos. En razón de aquello, este acontecimiento no puede ser integrado en ningún orden temático que pueda otorgarle un significado particular, pues precisamente la radical ausencia de sentido se posiciona como una de las cualidades fundamentales de este fenómeno. Así, esta experiencia en cuanto carece de significación tampoco puede ser justificada en función de un fin determinado, ya que en tanto inasumible ella resulta inintegrable en cualquier horizonte o contexto que busque explicarla. ${ }^{6}$ Por

resulta inconcebible pensar un tipo de experiencia en la que se encuentre ausente toda forma de intencionalidad, o sea, todo posible 'volverse' de la subjetividad como parece ser el caso del sufrimiento. De este modo, esta experiencia no solamente viene a poner en tensión la doctrina husserliana acerca de la atención, sino que junto con ello viene a cuestionar uno de los paradigmas fundamentales de la fenomenología elaborada por este autor.

5 “Que dans son phénomène propre, la souffrance soit inutile, qu'elle soit 'pour rien', est donc le moins qu'on puisse en dire".

${ }^{6}$ En relación con la ausencia de sentido que caracteriza el sufrimiento, Lévinas establece lo siguiente a propósito de la idea de 'teodicea' predominante en la filosofía moderna: "Reino de fines trascendentes, deseados por una sabiduría bienaventurada, por la bondad absoluta de un Dios definido, en cierta manera, por esta bondad sobrenatural, o bondad expandida, invisible, en la Naturaleza y en la Historia en la que ella comanda sus caminos, ciertamente dolorosos pero conducentes al Bien. Dolor que a partir de ahora tiene sentido, subordinado de una manera u otra a la finalidad metafísica entrevista por la fe o por la creencia en el progreso. Creencias presupuesta por la teodicea [...] Perspectivas suprasensibles para entrever en el sufrimiento, esencialmente gratuito y absurdo y, aparentemente arbitrario, una significación y un orden" (1991 106). De acuerdo con esto, si la descripción moderna de la 'teodicea' mienta aquella idea que justifica los males evidenciados sensiblemente por los individuos en función de un fin suprasensible como es el caso de la idea de 'salvación'; por su parte, la descripción levinasiana del sufrimiento como una 
este motivo, para quien se interroga preguntas tales como ¿qué significado tiene esta experiencia? o ¿por qué se sufre?, ellas resultan incontestables, ya que en esta situación el sujeto sufriente se descubre a sí mismo abandonado a un fenómeno que lo desborda absolutamente.

Resumiendo, las ideas expuestas hasta aquí, podemos realizar dos afirmaciones. Primero, las sensaciones mediante las que se anuncia el sufrimiento comportan tal exceso que hacen imposible toda síntesis, razón por la que si hay algo que caracteriza a esta experiencia es su carencia de sentido. Segundo, y producto de tal exceso característico de este fenómeno, podemos describirlo como una experiencia que torna imposible toda acogida o recepción, pues en ésta la conciencia en lugar de otorgar una forma precisa al acontecimiento que vivencia, asiste impotente al desgarramiento de sus propias capacidades. Hecha esta afirmación, algunas de las preguntas que pueden surgir son: 1) ¿qué tipo de relación se halla en juego en este fenómeno? O en otra formulación, ¿de qué modo hacemos experiencia de este suceso? Y 2) ¿qué comprensión de la sensibilidad y, por extensión, de la corporalidad se anuncia en esta vivencia? Con el objeto de resolver ambas interrogantes es menester describir la pasividad radical a través de la que Lévinas caracteriza esta experiencia.

\section{La irremisibilidad del sufrimiento como pasividad radical}

En La souffrance inutile podemos leer lo siguiente: "La pasividad del sufrimiento es más profundamente pasiva que la receptividad de nuestros sentidos, que es ya actividad de acogida." (1991 101)7. De acuerdo con esto, podemos señalar que el sufrimiento mienta una experiencia corporal en la que a un sujeto se le revela su propia existencia bajo el modo de la conmoción (commotio), vale decir, mediante un conjunto de sensaciones en la que ella se manifiesta profundamente alterada. En otras palabras, si nos preguntamos ¿qué es lo que se anuncia sensiblemente en este acontecimiento?, la respuesta no es sino nuestra misma existencia, de la que si bien no podemos hacer acogida o recepción alguna, no por ello nos resulta un hecho ajeno del que podamos guardar distancia. Precisando este punto, el sufrimiento en tanto posee un carácter corporal nos resulta irremisible, por lo que no podemos

experiencia que carece de toda significación, nos permite situar esta experiencia fuera de todo orden, contexto o fin que busque otorgarle un sentido determinado que posibilite su justificación. En otras palabras, el autor al posicionar el sufrimiento fuera de todo marco de significación, viene a poner en cuestión tanto la 'teodicea' como todas aquellas ideas que, buscando darle un sentido a esta vivencia permitan explicarla. Así las cosas, esta experiencia puede ser descrita de entrada como aquella que desborda todo posible 'horizonte hermenéutico' que busque comprenderla.

7 "La passivité de la souffrance est plus profondément passive que la réceptivité de nos sens qui est déjà activité de l'accueil". 
hacer oídos sordos ante esta vivencia $\mathrm{u}$ omitirla, pues en tanto evidenciada en carne propia afecta íntegramente a quien la padece. Con el objeto de aclarar este esquema, podemos pensar en formas de sufrimiento tales como el hambre o la depresión, en las que nuestra existencia se anuncia como un hecho insoportable cuya efectividad no podemos omitir. En Le temps et l'autre Lévinas nos dice que "el sufrimiento físico es, en todos sus grados, una imposibilidad de separarse del instante de la existencia. Ella es la irremisibilidad misma del ser. El contenido del sufrimiento se confunde con la imposibilidad de alejar de sí el sufrimiento" (1983 $55)^{8}$.

Así, si llevamos aun más lejos la reflexión del autor, podemos afirmar que la radicalidad de esta vivencia reside en última instancia en el hecho de no poder dejar de sufrir. De este modo, podemos sostener que un sujeto sufriente es en último término, aquel que sufre su propio sufrimiento en tanto incapaz de claudicar de dicha experiencia. Por estas razones, este fenómeno al tiempo que revela una ausencia de sentido y la irremisibilidad de nuestra existencia, pone en evidencia otro elemento, a saber, la pasividad radical que lo acompaña. Aclarando esta idea, el sufrimiento mienta un acontecimiento absolutamente pasivo en un doble sentido; primero, en la medida que se da a nuestro pesar y, segundo, que por mucho que se produzca contra nuestra voluntad, somos incapaces de sustraernos a él. En este sentido, esta vivencia no hace referencia meramente a una sensación de desagrado, como por ejemplo, cuando evidenciamos un olor que nos disgusta, ya que la situación en este caso es más extrema aun, al punto que en ella resulta imposible huir o protegerse del padecimiento que nos afecta. En esta misma línea y tal como establece Roberto Walton esta vivencia representa "una carga inherente a la vida, en la medida en que ella se experimenta a sí misma como sufrimiento, y su peso se torna insoportable en razón de que es algo de lo que es imposible librarse" (2016 29).

De esta manera, el sufrimiento pone en relieve la vulnerabilidad de la subjetividad que lo experimenta, es decir, la posibilidad de ser herido (vulnus) y conmocionado que se anuncia en la incapacidad de protegerse de él. Así, si intentamos reconstruir este fenómeno nos encontramos en él con una subjetividad absolutamente abandonada ${ }^{9}$, o sea, arrojada a una experiencia en la que todo

\footnotetext{
8 "la souffrance physique, à tous ses degrés, est une impossibilité de se détacher de l'instant de l'existence. Elle est l'irrémissibilité même de l'être. Le contenu de la souffrance se confond avec l'impossibilité de se détacher de la souffrance".

9 En relación con el 'abandono' que es puesto en evidencia en experiencias tales como el sufrimiento, Lévinas nos dice que: “La derelicción, el abandono a las posibilidades impuestas, da a la existencia humana un carácter de hecho en un sentido muy fuerte y dramático del término: es un hecho que se comprende como tal mediante su efectividad" (2016 99). Si seguimos este pasaje, podemos establecer que el carácter de abandono por medio del que se nos revela esta vivencia,
} 
posible repliegue o cuidado de sí resulta impensable, ya que ésta última se descubre a sí misma en la intemperie.

Dicho esto, si intentamos vincular las reflexiones recién expuestas con los elementos presentados en la primera parte de este texto, podemos afirmar que en el sufrimiento se pone en relieve una doble ausencia de horizonte; de una parte, ausencia de un horizonte hermenéutico que permita dar sentido o explicar esta experiencia y; de otra, ausencia de un horizonte de posibilidades en tanto el sujeto que padece esta vivencia se revela a sí mismo como absolutamente impotente ante aquel acontecimiento que desgarra su existencia. En suma, en el sufrimiento toda consolación o alivio resulta inconcebible, dado que en esta vivencia toda esperanza futura es borrada por una situación irremisible a la que estamos clavados en el presente.

Ahora bien, el cuestionamiento hacia el que nos conduce ineludiblemente esta descripción del sufrimiento es ¿cuál es el soporte de la vulnerabilidad que deja de manifiesto esta experiencia? Si respondemos esta inquietud con Lévinas, podemos indicar que el fundamento de dicha vulnerabilidad reside indudablemente en la dimensión corporal mediante la que se anuncia esta vivencia. En otras palabras, el sufrimiento en la medida que mienta una experiencia evidenciada en cuerpo propio nos impide mantener distancia o resguardo alguno frente a él, razón por la que, el sujeto sufriente al tiempo que descubre su existencia como un acontecimiento inasumible, se revela a sí mismo como una subjetividad encarnada, esto es, inseparable de su propio cuerpo.

De este modo, en tanto somos sujetos encarnados, todo aquello que afecta a nuestro cuerpo simultáneamente nos afecta a nosotros. En función de esto, experiencias tales como el cansancio o la fatiga no ponen sino en evidencia el hecho de que nuestra existencia íntegra se encuentra cansada o fatigada respectivamente, o, en el caso del sufrimiento, que es ella la que padece su propia conmoción. Por esto, es a causa de nuestra condición corporal que toda forma de protección o cuidado resulta inconcebible a la de hora de describir a la subjetividad, puesto que ella en tanto inseparable de su cuerpo se encuentra expuesta a diversos padecimientos concretos y materiales que de modo constante alteran su existencia. Así, si volvemos a la inquietud relativa a la vulnerabilidad que pone en relieve este fenómeno, podemos afirmar que ella encuentra su fundamento en nuestra

pone en relieve un tipo de experiencia hacia la que, antes de 'volvernos' activamente, nos descubrimos entregados pasivamente. En este sentido, la radicalidad del sufrimiento es tal que en esta experiencia no solamente no podemos volvernos hacia ella intencionalmente, sino que además nos encontramos abandonados a un 'hecho' irremisible del que no podemos desvincularnos de ninguna manera. Así, para quien sufre la propia 'existencia' -que se anuncia en dicha experienciadeviene un fenómeno ineludible del cual no se puede claudicar. 
corporalidad, al tiempo que esta última devela cierta comprensión fenomenológica de la sensibilidad en la que ella es entendida precisamente como vulnerabilidad, es decir, como la posibilidad de ser afectados o heridos por todo aquello que se nos presenta. Lévinas nos dice que "en el sufrimiento, la sensibilidad es vulnerabilidad más pasiva que la receptividad" (1991 101) ${ }^{10}$, motivo por el que podemos sostener que la pasividad y vulnerabilidad que manifiesta esta experiencia no pueden ser comprendidas como la contracara de la actividad, ni tampoco como una pasividad en la actividad, ya que en esta situación de aguda impotencia lo que se encuentra ausente precisamente es toda posible capacidad que permita a un sujeto sustraerse a la misma.

En este sentido, "la humanidad del hombre que sufre se halla abrumada por el mal que la desgarra" (1991 101 $)^{11}$ al punto que, podemos calificar esta experiencia como aquella en la que un individuo pierde su propia humanidad. En otros términos, tal es la magnitud de este fenómeno que quién lo experimenta se deshumaniza pues pierde todo poder o capacidad al estar clavado a una existencia insoportable que no puede abandonar.

Considerada esta explicación, podemos afirmar que el sufrimiento nos atrapa a tal punto que incluso podemos cuestionarnos si somos efectivamente nosotros los sujetos de aquella experiencia que nos desespera en cuanto incapaces de encontrar una salida. Resumiendo este punto, podemos sostener que mientras la teoría husserliana de la atención nos invita a pensar nuestra sensibilidad como recepción o acogida, por su parte Lévinas al conducirnos a pensar el sufrimiento no solamente muestra la no pertinencia de aquel modelo para explicar esta experiencia, sino que además resignifica el carácter mismo de la sensibilidad al movernos a comprenderla ya no como receptividad sino como vulnerabilidad, es decir, como pasividad absoluta.

Siguiendo esta línea, podemos indicar que en la experiencia del sufrimiento la afección primera mediante la cual se anuncia esta vivencia posee tal magnitud que desgarra toda posible síntesis o actividad que, fundándose sobre ella posibilite la mirada teórica de la conciencia $y$, con ello, el conocimiento. En términos equivalentes, la afección que está en juego en este fenómeno posee tal grado que en ella la sensibilidad no se posiciona ya como el fundamento de la actividad intencional de la subjetividad sino como vulnerabilidad, es decir, como un acontecimiento que desgarra la existencia misma de quien la evidencia. Por este motivo, Renaud Barbaras afirma que "la herida que hay en la vulnerabilidad es más profunda ya que ella concierne en el fondo la integridad misma del sujeto que

\footnotetext{
10 "Dans la souffrance la sensibilité est vulnérabilité, plus passive que la réceptivité".

11 "L'humanité de l'homme qui souffre est accablée par le mal qui la déchire".
} 
es afectado: ser vulnerable es ser amenazado en su integridad y finalmente en su existencia misma" (2018 48) $)^{12}$.

Así las cosas, la afección propia del sufrimiento viene a romper con su irrupción toda comprensión de nuestra experiencia mediante la lógica de la acogida o la recepción al tiempo que, revela nuestra existencia como un hecho irremisible al que nos descubrimos abandonados y del que, no obstante, no podemos declinar.

\section{Conclusión}

Para finalizar esta indagación, quiero poner en evidencia algunas de las principales notas que a mi parecer ha dejado en claro este intento de elaborar una fenomenología del sufrimiento.

En primer orden, esta experiencia se nos revela como aquella en que resulta imposible toda actividad de acogida o recepción por parte de la subjetividad que la evidencia, o sea, todo intento que busque integrar esta experiencia dentro de un determinado horizonte de sentido. De acuerdo con esto, el sufrimiento al poner en tensión toda acogida o recepción sobre la cual pueda montarse la actividad intencional de la conciencia, nos invita a pensar nuestra experiencia fuera del marco atencional por medio del que la describe Husserl, es decir, ya no bajo el modo de un volverse sobre una determinada vivencia, sino más bien mediante un descubrirse abandonado a ella.

En segundo orden, esta vivencia se anuncia mediante su carácter irremisible, vale decir, a partir de la imposibilidad de sustraerse a ella, motivo por el que podemos sostener que la complejidad de esta experiencia reside en el hecho que en ésta no sólo es imposible toda acogida, sino además toda salida o evasión que nos permita desvincularnos de ella.

En tercer orden, este fenómeno pone en relieve cierta comprensión de la sensibilidad en la que ésta se revela como vulnerabilidad, es decir, como cierta forma de pasividad radical en la que producto del carácter sensible de nuestra existencia, nos descubrimos expuestos a diversos padecimientos que de modo constante la alteran íntegramente. Estos tres elementos recién mencionados, dejan en claro la que desde mi punto de vista es la principal conclusión de este trabajo, a saber, que un análisis fenomenológico del sufrimiento no solamente nos convoca a redefinir nociones tales como la corporalidad y la sensibilidad, sino que en último término, nos mueve a repensar el carácter mismo de nuestra subjetividad.

\footnotetext{
12 "la blessure dont il y va dans la vulnérabilité est très profonde car elle concerne au fond l'intégrité même du sujet qui est atteint: être vulnérable, c'est être menacé dans son intégrité et finalement dans son existence même."
} 


\section{Bibliografía}

Barbaras, Renaud. "Finitude et vulnérabilité". Vulnérabilité et empathie. Approches phénoménologiques. Ed. Élodie Boublil. Paris: Hermann Philosophie, 2018. 4762

Husserl, Edmund. Experiencia y Juicio. México: UNAM, 1980.

Husserl, Edmund. Meditaciones Cartesianas. México: Fondo de Cultura Económica, 2005.

Lévinas, Emmanuel. Le temps et l'autre. Paris: PUF, 1983.

Lévinas, Emmanuel. Entre nous. Essais sur le penser-à-l'autre. Paris: Le livre de poche, 1991.

Lévinas, Emmanuel. En découvrant l'existence avec Husserl et Heidegger. Paris: Vrin, 2016.

Lévinas, Emmanuel. Totalité et infini. Essai sur l'extériorité. Paris: Le livre de poche, 1971.

Mate, Manuel Reyes. El tiempo, tribunal de la historia. Madrid: Trotta, 2018.

Osswald, Andrés. "Fenomenología de la sensación. Un estudio sobre los Analysen zur passiven Synthesis de Husserl", THÉMATA: Revista de Filosofía 56 (2017): 61-82.

Walton, Roberto. "Autoafección y acontecimiento". Problemas de fenomenología material. Eds. Mario Lipsitz y Carlos Belvedere. Buenos Aires: Ediciones UNGS, 2016. 25-42. 